\title{
Thick-disc model to explain the spectral state transition in NGC 247
}

\author{
Jing Guo, ${ }^{1}$, Mouyuan Sun, ${ }^{2,3 \star ~ W e i-M i n ~ G u, ~}{ }^{1} \dagger$ Tuan Yi, ${ }^{1}$ \\ ${ }^{1}$ Department of Astronomy, Xiamen University, Xiamen, Fujian 361005, China \\ ${ }^{2}$ CAS Key Laboratory for Research in Galaxies and Cosmology, Department of Astronomy, \\ University of Science and Technology of China, Hefei 230026, China \\ ${ }^{3}$ School of Astronomy and Space Science, University of Science and Technology of China, Hefei 230026, China
}

Accepted XXX. Received YYY; in original form ZZZ

\begin{abstract}
We propose the thick-disc model of Gu et al. (2016) to interpret the transition between soft ultraluminous state (SUL) and supersoft ultraluminous (SSUL) state in NGC 247. As accretion rate increases, the inner disc will puff up and act as shield to block the innermost X-ray emission regions and absorb both soft and hard X-ray photons. The absorbed X-ray emission will be re-radiated as a much softer blackbody X-ray spectrum. Hence NGC 247 shows flux dips in the hard X-ray band and transits from the SUL state to the SSUL state. The $\sim 200$ s transition timescale can be explained by the viscous timescale. According to our model, the inner disc in the super-soft state is thicker and has smaller viscous timescale than in the soft state. X-ray flux variability, which is assumed to be driven by accretion rate fluctuations, might be viscous time-scale invariant. Therefore, in the SSUL state, NGC 247 is more variable. The bolometric luminosity is saturated in the thick disc; the observed radius-temperature relation can therefore be naturally explained.
\end{abstract}

Key words: accretion, accretion discs - binaries: close — black hole physics galaxies: individual (NGC 247)

\section{INTRODUCTION}

Typical ultraluminous X-ray sources (ULXs) are nonnuclear accreting X-ray sources whose luminosity exceeds the Eddington luminosity (for review, see Di Stefano \& Kong 2003; Feng \& Soria 2011; Kaaret et al. 2017). Nevertheless, their physical properties remain unclear since being detected for 40 years (Fabbiano 1989). It is likely that ULXs are powered by super-Eddington accretion onto stellar-mass black holes (Gladstone \& Roberts 2009). A few ULXs are considered as intermediate-mass black holes candidates, like HLX1 (Farrell et al. 2009; Godet et al. 2009; Sun et al. 2016), or neutron stars (Bachetti et al. 2014). There is a special subclass of ULXs that show very soft spectra, namely, ultraluminous supersoft X-ray sources (ULSs) (Di Stefano et al. 2004).

Two ULX/ULS unification models, which are based on super-Eddington accretion onto stellar-mass black hole, are proposed (Urquhart \& Soria 2016; Gu et al. 2016). In both scenarios, the compact X-ray emission regions are obscured by high column density gas. In the first model, powerful out-

^ E-mail: ericsun@ustc.edu.cn

† E-mail: guwm@xmu.edu.cn flows can block our line of sight (Urquhart \& Soria 2016). In the second model, Gu et al. (2016) argued that, for super Eddington ratio sources, the accretion disc is geometrically thick and acts as a "shielding" gas (a similar scenario is also proposed to explain weak-line quasars; see Wu et al. 2011; Luo et al. 2015). Therefore, at certain inclination angles, hard X-ray photons are absorbed and thermalized; these ULXs appear as ULSs. Though with different mechanisms, the two models argue that ULSs tend to be more edge-on and have larger accretion rates compared with ULXs.

ULXs are not stationary systems. On the contrary, these sources suffer significant X-ray variability (e.g., Kubota et al. 2001; Feng \& Kaaret 2006; Roberts et al. 2006; Kaaret \& Feng 2009; Weng \& Feng 2018). It is possible that a ULX can transit to ULS and vice versa. Indeed, by analyzing data of XMM-Newton, Chandra, Swift and Hubble Space Telescope observations, Feng et al. (2016) first found the transitions between the supersoft ultraluminous (SSUL) regime and the soft ultraluminous regime (SUL) in the spectra of NGC 247. In the SSUL regime, the X-ray spectrum of NGC 247 can be well characterized by a cool (i.e., the temperature $T \lesssim 0.1$ $\mathrm{keV}$ ) blackbody component which is similar to other ULSs. For the first time, the X-ray spectrum of NGC 247 in the SUL regime shows a strong power-law component with flux 
comparable to the soft blackbody emission. Therefore, as pointed out by Feng et al. (2016), NGC 247 might not always be classified as a ULS and they use SSUL and SUL to refer to this source. Its hard spectrum in the SUL regime might be similar to that of a ULX.

The observational facts of the SUL/SSUL transition can be summarized as follows.

1. as NGC 247 enters the SSUL regime, the $0.2-10 \mathrm{keV}$ $\mathrm{X}$-ray flux decreases.

2 . the transition timescale is $\sim 200 \mathrm{~s}$.

3. the blackbody temperature and the radius are anticorrelated, i.e., $R_{\mathrm{bb}} \propto T_{\mathrm{bb}}^{-2.8 \pm 0.3}$.

4. the X-ray flux is more variable in SSUL than in SUL.

Feng et al. (2016) argued that their results can be explained by the outflow model. In this work, we propose an alternative model which is base on $\mathrm{Gu}$ et al. (2016) to explain the observed characters in NGC 247. This paper is formatted as follows. In Section 2, we introduce our thick-disc model. In Section 3, we make conclusions and discussion.

\section{THICK-DISC MODEL}

We propose the thick-disc model to explain the SUL/SSUL transition (see Fig. 1). In our model, the accretion disc can be divided into two parts. The inner part of the disc puffs up because of super-Eddington accretion (Abramowicz et al. 1988; Gu et al. 2009; Gu 2012). Such geometrical structure is also confirmed by recent numerical simulations (Narayan et al. 2016). Meanwhile, significant outflows are well expected (see, e.g., Ohsuga \& Mineshige 2011; Gu 2015; Sądowski $\&$ Narayan 2015). The outer part (i.e., $R \gtrsim R_{\mathrm{tr}}$ ) can be described by the classical thin disc theory (Shakura \& Sunyaev 1973). In our model, the hot innermost region could emit hard X-ray photons. If the inclination angle is small enough (i.e., more face-on), we can directly detect hard X-ray emission from the innermost regions. On the other hand, if the inclination is not small (i.e., more edge-on), most of hard and soft X-ray photons could be obscured by the thick disc. Therefore, we can only detect much softer X-ray photons that are produced in the effective absorption photosphere. The critical inclination angle $\theta_{c}$ should depend on the vertical structure of the thick disk. According to the numerical simulations of Narayan et al. (2016), for the case of $\dot{m}=11$, $\theta_{c} \sim 25^{\circ}$, which is adopted by Gu et al. (2016).

In the SUL regime (i.e., the star symbol in Fig. 2) of NGC 247, our line of sight is assumed to be close to the edge of the thick disc (i.e., the left panel of Fig. 1). As accretion rate increases (i.e., the triangle symbol in Fig. 2), the inner part of the accretion disc will puff up into an even thicker one and block our line of sight (i.e., the right panel of Fig. 1) and vice versa. The puffed-up disc can absorb innermost X-ray emission and re-radiate a much softer blackbody X-ray spectrum from its photosphere. Therefore, NGC 247 undergoes a transition between SUL and SSUL.

In the SSUL regime, we expect that the $0.2-10 \mathrm{keV}$ $\mathrm{X}$-ray flux to be suppressed since the innermost region is blocked by the thick disc. Such suppression increases with increasing energy, which is consistent with the observational fact \#1 (see fig. 1 of Feng et al. 2016).

The transition timescale should be the viscous timescale

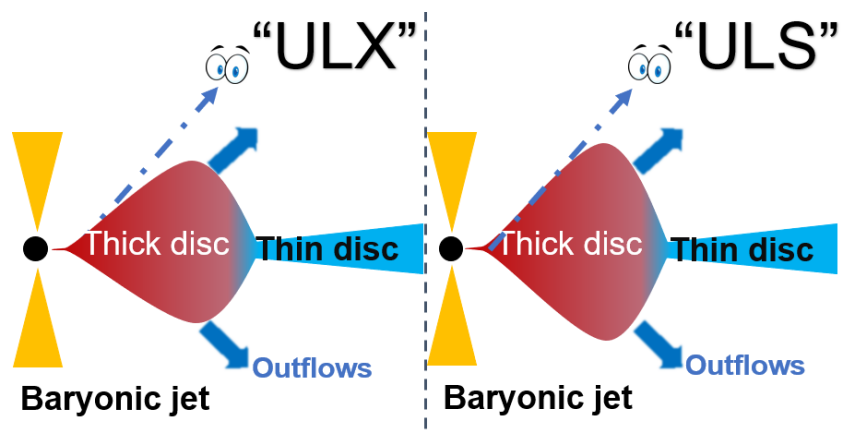

Figure 1. Illustration of the thick-disc model of Gu et al. 2016. With sufficiently high accretion rate, the inner disc puffs up while the outer one remains geometrically thin. Significant outflows are also expected; the baryonic jet is launched near the BH which is responsible for the highly blueshifted emission lines (Liu et al. 2015). If the inclination angle is small (i.e., $\lesssim 25^{\circ}$ ), we can directly observe the hard X-ray emission from the innermost regions; the source is identified as an "ULX". As accretion rate increases, the inner disc is thicker; the thicker disc blocks our view of the innermost X-ray regions. We instead only detect very soft X-ray emission produced from the photosphere. Hence, the source appears as an "ULS".

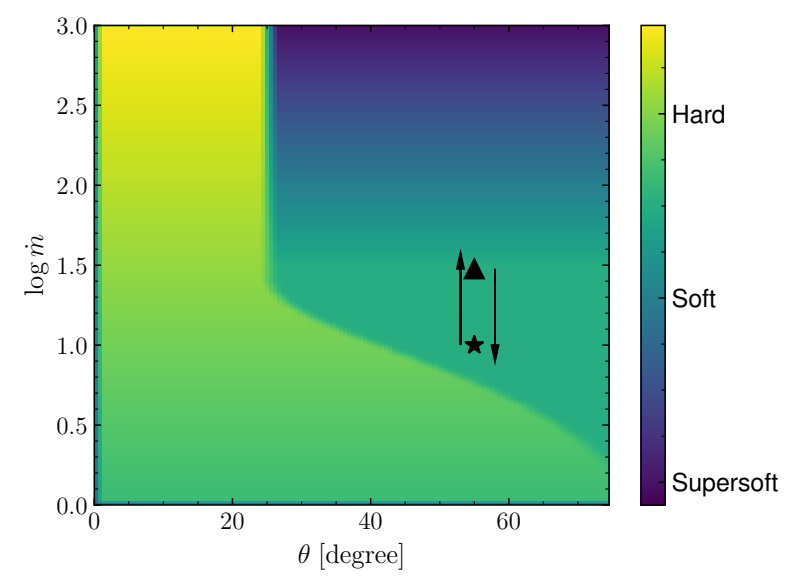

Figure 2. Illustration of the unification of different spectral types of ULXs. Qualitatively, the star represent the SSUL regime, and the triangle represent the SUL regime. For a given source (e.g., NGC 247), the inclination angle $\left(\theta \gtrsim 25^{\circ}\right)$ is expected to be fixed. However, the accretion rate can vary significantly on viscous timescales. When the accretion rate fluctuates, the transitions between SSUL and SUL occur.

(see Eqs. 5.68 and 5.89 of Frank et al. (2002))

$t_{\mathrm{vis}} \sim 110 \frac{0.01}{\alpha}\left(\frac{R}{H}\right)^{2}\left(\frac{M_{\mathrm{BH}}}{23 M_{\odot}}\right)^{-\frac{1}{2}}\left(\frac{R}{1.4 \times 10^{9} \mathrm{~cm}}\right)^{\frac{3}{2}} \mathrm{~s}$

where $\alpha, M_{\mathrm{BH}}, H$ and $R$ are the dimensionless viscosity parameter, the black hole mass, the height of thick disc and the transition radius, respectively. For geometrically thick disc, we can use $R / H \sim 1$.

The exact value of the black hole mass of NGC 247 


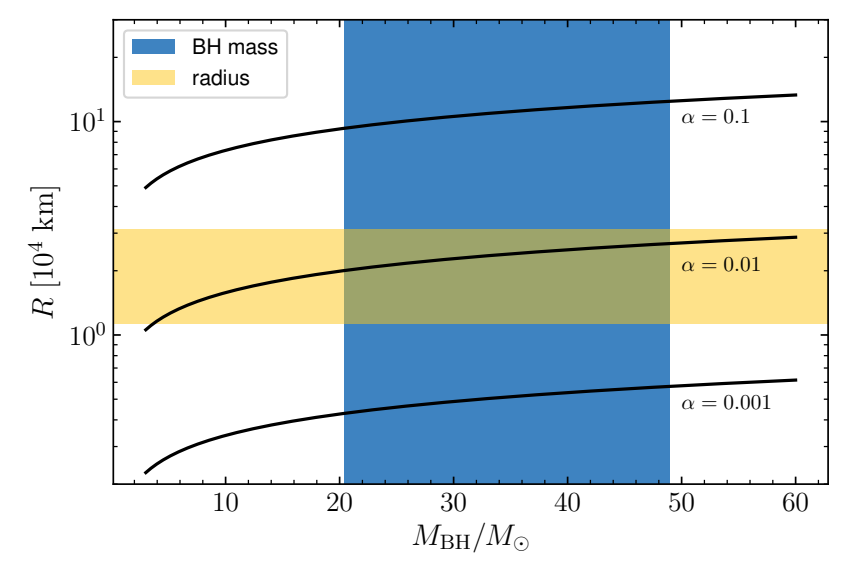

Figure 3. The allowed parameter space for NGC 247. The blue shaded region is for the $16 \mathrm{th}-84$ th percentiles of the ten inferred $M_{\mathrm{BH}}$ (i.e., by adopting $\left.L_{\mathrm{bol}}=1.7 L_{\mathrm{Edd}}\right)$. The yellow shaded region corresponds to the $16 \mathrm{th}-84 \mathrm{th}$ percentiles of the ten estimates of $R_{\mathrm{tr}}$ (Feng et al. 2016). The three solid lines indicate the relation between $R_{\mathrm{tr}}$ and $M_{\mathrm{BH}}$ if the transition timescale (i.e., $\sim 200 \mathrm{~s}$ ) corresponds to the viscous timescale at $R_{\operatorname{tr}}$ (i.e., Eq. 1).

remains unknown. In the thick disc model of $\mathrm{Gu}$ et al. (2016), the bolometric luminosity $L_{\mathrm{bol}} \sim 1.7 L_{\mathrm{Edd}}=2.14 \times$ $10^{38}\left(M_{\mathrm{BH}} / M_{\odot}\right) \mathrm{erg} \mathrm{s}^{-1}$. We can use this relation to infer $M_{\mathrm{BH}}$. NGC 247 is a highly variable source, therefore the inferred $M_{\mathrm{BH}}$ changes for the ten observations. The 16th-84th percentiles of the ten inferred $M_{\mathrm{BH}}$ are shown as the blue shaded region in Fig. 3. Meanwhile, $R_{\mathrm{tr}}$ can be constrained by fitting the ten X-ray spectra (Feng et al. 2016); the 16th84th percentiles of the ten estimates of $R_{\mathrm{tr}}$ are shown as the yellow shaded region of Fig. 3. The overlapping region is the allowed parameter space for NGC 247.

According to our model, the viscous timescale at $R_{\mathrm{tr}}$ (i.e., Eq. 1) should be $\sim 200 \mathrm{~s}$ (see the observational fact $\# 2$ ). If so, the required $R_{\mathrm{tr}}-M_{\mathrm{BH}}$ relations for different choices of $\alpha$ are shown as solid lines in Fig. 3. It is evident that, if $\alpha \sim 0.01$, our model can naturally explain the observed $200 \mathrm{~s}$ transition timescale. The dimensionless viscosity parameter $\alpha$ is determined by the magnetorotational instability turbulence and can only be inferred from MHD numerical simulations or observations. Observationally, $\alpha \sim 0.1$ is estimated from outbursts of Dwarf nova and X-ray transients (King et al. 2007). However, MHD simulations seem prefer a much lower $\alpha \sim 0.01$ even in the radiation-pressure dominated regions (for a summary of the value of $\alpha$ in MHD simulations, see figure 1 of Blaes 2014, and references therein).

For NGC 247, the radius-temperature relation is $R_{\mathrm{bb}} \propto$ $T_{\mathrm{bb}}^{-2.8 \pm 0.3}$. According to our thick-disc model, $R_{\mathrm{bb}}$ scales as $T_{\mathrm{bb}}^{-2}$. Therefore, our model and the data are consistent within the $3 \sigma$ uncertainty.

X-ray variability might be caused by the fluctuation of accretion rate (Lyubarskii 1997). If so, it is natural to expect that a viscous time-scale invariant $\mathrm{X}$-ray variability. In the SSUL regime, the disc is thicker than that of the SUL regime. That is, the viscous timescale is smaller in the SSUL regime. Therefore, for fixed observational timescales, the $\mathrm{X}$ ray flux of NGC 247 is more variable in the SSUL regime.
In addition, if $\dot{m}$ increases to $\sim 115$, the blackbody temperature would decrease to be less than $50 \mathrm{eV}$ (Eq. (5) of Gu et al. 2016). As a consequence, such sources become invisible to X-ray telescopes (e.g., Chandra) and show extreme X-ray variability and transient behaviours.

\section{CONCLUSIONS AND DISCUSSION}

In this paper, we propose the thick-disc model of $\mathrm{Gu}$ et al. (2016) to interpret the observational facts of NGC 247. As the accretion rate fluctuates, the thickness of the puffed-up disc changes. As a result, the puffed-up disc can block or leave our line-of-sight. Such a model can explain the transition between SUL and SSUL regimes in NGC 247.

As pointed out by Feng et al. (2016), the outflow model can also explain some observational aspects of NGC 247, including the $R_{\mathrm{bb}}-T_{\mathrm{bb}}$ relation. The $\sim 200 \mathrm{~s}$ timescale and variability might be driven by some instabilities and/or turbulence in the outflow; alternatively, the disc instabilities we mentioned in Section 2 might also induce some variations of outflow properties. So far, there is no solid evidence for or against the outflow model or our thick-disc model.

Compared with the outflow model, the required accretion rate in our model is much lower. To illustrate this fact, we estimate the accretion rate via Eq. (5) of Gu et al. (2016) and find that $\dot{m} \sim 10-30$, which is one order of magnitude lower than that of the outflow model (for NGC 247, see fig. 5 of Feng et al. 2016).

With such high accretion rates, powerful outflows are inevitable both from theoretical considerations (e.g., Weng \& Zhang 2011; Gu 2015) and numerical simulations (e.g., Ohsuga \& Mineshige 2011; Sa̧dowski \& Narayan 2015). Meanwhile, blueshifted emission lines are also observed in some ULXs (Pinto et al. 2016; Walton et al. 2016). In principle, such outflows might also act as shield and increase our line-of-sight optical depth. It is quite possible that both the thick disc and the outflows play significant roles in obscuring the inner X-ray emission regions (see Fig. 1). If so, the critical accretion rate for the SSUL regime could be even lower (Gu et al. 2016).

In future, our thick-disc model should be observationally distinguishable from the outflow one since the required mass rate are quite different in the two models. We propose a few possible observational tests. First, the two models can be distinguished if we can measure the mass outflow rate. Both the two models predict massive outflows; however, the mass outflow rate of our thick-disc model is much lower than that of the outflow model. Outflow signatures, i.e., absorption/emission lines, are indeed detected in NGC 247, several other ULSs (Urquhart \& Soria 2016) and ULXs (Pinto et al. 2016; Walton et al. 2016; Kosec et al. 2018). According to our model, the lower mass rate outflows are responsible for the absorption features (around $1 \mathrm{keV}$ ). If the mass rate of such outflow can be inferred from these features, our model and the outflow model can be distinguished. The low signalto-noise data prevent us from constraining the mass outflow rate. Future facilities, e.g., $H U B s^{1}$ and Athena (Nandra et

1 For details, please refer to http://heat.tsinghua.edu.cn/ ^hubs/en/index.html 
al. 2013), can provide high-resolution soft X-ray spectra and might be able to measure the mass outflow rate. In addition, huge bubbles are observed around some ULXs (e.g., Kaaret et al. 2004; Kaaret \& Corbel 2009; Moon et al. 2011; Cseh et al. 2012); if such bubbles are inflated by outflows, we might also be able to estimate the mass outflow rate by considering their ages, kinematics and total energies (e.g., Pakull et al. 2006; Siwek et al. 2017). However, similar bubbles are not observed for ULSs. Future possible discoveries of ULS bubbles would be very interesting and can verify the ULS models. The two models can also be tested by the intrinsic fraction of ULSs to ULXs. According to both models, ULSs tend to have larger accretion rate and higher inclination angle with respect to ULXs. The accretion rate distribution for ULXs can be inferred from binary population synthesis models (e.g., Madhusudhan et al. 2008; Pavlovskii et al. 2017); the distribution drops rapidly at high accretion rate end. If so, our thick-disc model predicts a higher fraction of ULSs to ULXs than that of the outflow model because the required accretion rate to be an ULS in our model is roughly one order of magnitude lower. If we adopt the accretion rate distribution of figure 10 of Madhusudhan et al. (2008) for stellar black holes (by assuming an average $M_{\mathrm{BH}}=10 M_{\odot}$ and the radiative efficiency of 0.1 ), the ratio of the fraction of systems with $\dot{m}>30$ to those with $30>\dot{m}>1$ is $1 / 32$. If we take this ratio and the fact that the viewing angle of an ULS must be larger than 25 degree, the expected ratio of number of ULS to that of ULX is about $1 / 45$. This ratio is roughly consistent with the observed one, albeit with large uncertainties in both theoretical and observational (because the sample size of ULS is quite small) ones. All in all, future observations of ULSs can decipher the super-Eddington accretion physics.

\section{ACKNOWLEDGEMENTS}

We thank the anonymous referee for his/her helpful comments that improved the paper. We are grateful to Hua Feng, Xiangdong Li, Dabin Lin and Yaping Li for valuable discussion. This work was supported by the National Natural Science Foundation of China under grants 11573023, 11333004 and 11603022. M.Y.S. acknowledges the support from the China Postdoctoral Science Foundation (2016M600485).

\section{REFERENCES}

Abramowicz, M. A., Czerny, B., Lasota, J. P., \& Szuszkiewicz, E. 1988, ApJ, 332, 646

Bachetti, M., Harrison, F. A., Walton, D. J., et al. 2014, Nature, 514,202

Blaes, O. 2014, Space Sci. Rev., 183, 21

Cseh, D., Corbel, S., Kaaret, P., et al. 2012, ApJ, 749, 17

Di Stefano, R., \& Kong, A. K. H. 2003, ApJ, 592, 884

Di Stefano, R., Kong, A. K. H., Greiner, J., et al. 2004, ApJ, 610, 247

Fabbiano, G. 1989, ARA\&A, 27, 87

Farrell, S. A., Webb, N. A., Barret, D., Godet, O., \& Rodrigues, J. M. 2009, Nature, 460, 73

Feng, H., \& Kaaret, P. 2006, ApJ, 650, L75

Feng, H., \& Soria, R. 2011, New Astron. Rev., 55, 166

Feng, H., Tao, L., Kaaret, P., \& Grisé, F. 2016, ApJ, 831, 117
Frank, J., King, A., \& Raine, D. J. 2002, Accretion Power in Astrophysics, by Juhan Frank and Andrew King and Derek Raine, pp. 398. ISBN 0521620538. Cambridge, UK: Cambridge University Press, February 2002., 398

Gladstone, J. C., \& Roberts, T. P. 2009, MNRAS, 397, 124

Godet, O., Barret, D., Webb, N. A., Farrell, S. A., \& Gehrels, N. 2009, ApJ, 705, L109

Gu, W.-M. 2012, ApJ, 753, 118

Gu, W.-M. 2015, ApJ, 799, 71

Gu, W.-M., Sun, M.-Y., Lu, Y.-J., Yuan, F., \& Liu, J.-F. 2016, ApJ, 818, L4

Gu, W.-M., Xue, L., Liu, T., \& Lu, J.-F. 2009, PASJ, 61, 1313

Kaaret, P., \& Corbel, S. 2009, ApJ, 697, 950

Kaaret, P., \& Feng, H. 2009, ApJ, 702, 1679

Kaaret, P., Feng, H., \& Roberts, T. P. 2017, ARA\&A, 55, 303

Kaaret, P., Ward, M. J., \& Zezas, A. 2004, MNRAS, 351, L83

King, A. R., Pringle, J. E., \& Livio, M. 2007, MNRAS, 376, 1740

Kubota, A., Makishima, K., \& Ebisawa, K. 2001, ApJ, 560, L147

Kosec, P., Pinto, C., Fabian, A. C., \& Walton, D. J. 2018, MNRAS, 473, 5680

Liu, J.-F., Bai, Y., Wang, S., et al. 2015, Nature, 528, 108

Luo, B., Brandt, W. N., Hall, P. B., et al. 2015, ApJ, 805, 122

Lyubarskii, Y. E. 1997, MNRAS, 292, 679

Madhusudhan, N., Rappaport, S., Podsiadlowski, P., et al. 2008, ApJ, 688, 1235.

Moon, D.-S., Harrison, F. A., Cenko, S. B., \& Shariff, J. A. 2011, ApJ, 731, L32

Narayan, R., Zhu, Y., Psaltis, D., \& Saḑowski, A. 2016, MNRAS, 457,608

Nandra, K., Barret, D., Barcons, X., et al. 2013, ArXiv e-prints , arXiv:1306.2307.

Ohsuga, K., \& Mineshige, S. 2011, ApJ, 736, 2

Pakull, M. W., Grisé, F., \& Motch, C. 2006, Populations of High Energy Sources in Galaxies, 230, 293

Pavlovskii, K., Ivanova, N., Belczynski, K., \& Van, K. X. 2017, MNRAS, 465, 2092

Pinto, C., Middleton, M. J., \& Fabian, A. C. 2016, Nature, 533, 64

Roberts, T. P., Kilgard, R. E., Warwick, R. S., Goad, M. R., \& Ward, M. J. 2006, MNRAS, 371, 1877

Sądowski, A., \& Narayan, R. 2015, MNRAS, 453, 3213

Shakura, N. I., \& Sunyaev, R. A. 1973, A\&A, 24, 337

Siwek, M., Sądowski, A., Narayan, R., Roberts, T. P., \& Soria, R. 2017, MNRAS, 470, 361

Soria, R., \& Kong, A. 2016, MNRAS, 456, 1837

Sun, M., Gu, W.-M., Yan, Z., Wu, Q., \& Liu, T. 2016, MNRAS, 463, L99

Urquhart, R., \& Soria, R. 2016, MNRAS, 456, 1859

Walton, D. J., Fürst, F., Bachetti, M., et al. 2016, ApJ, 827, L13

Weng, S.-S., \& Feng, H. 2018, ApJ, 853, 115

Weng, S.-S., \& Zhang, S.-N. 2011, ApJ, 739, 42

Wu, J., Brandt, W. N., Hall, P. B., et al. 2011, ApJ, 736, 28 